JORNADAS

\title{
TRATAMIENTO FARMACOLÓGICO DE LA DIABETES MELLITUS PARA PACIENTES CON ENFERMEDAD RENAL
}

\author{
PHARMACOLOGICAL TREATMENT OF \\ DIABETES MELLITUS IN PATIENTS WITH KIDNEY DISEASE
}

María Florencia Aranguren

\begin{abstract}
RESUMEN
El manejo de la hiperglucemia en el paciente con enfermedad renal (ER) es complejo. El control estricto de la glucemia y los factores de riesgo pueden evitar la aparición y progresión de la ER; para esto es importante lograr metas glucémicas estrictas y la elección de drogas que disminuya la albuminuria y la caída del filtrado glomerular. Además en pacientes con ER debe considerarse que algunas drogas han demostrado un efecto directo sobre el riñón, más allá del descenso de la glucemia. Al momento del tratamiento, deberían tenerse en cuenta otros factores propios de la ER como mayor retención hidrosalina, riesgo cardiovascular, riesgo de hipoglucemias, mayor vida media de las drogas y el riesgo de fracturas, entre otros.
\end{abstract}

Palabras clave: enfermedad renal por diabetes, tratamiento no insulínico, insulinoterapia.

Revista de la Sociedad Argentina de Diabetes 2017; Vol. 51 (94-99)

\begin{abstract}
The management of hyperglycemia in the patient with kidney disease $(K D)$ is complex. The strict control of glycemia and risk factors, may avoid the onset and progression of $K D$, so it is important to achieve strict glycemic targets and select drugs that reduce albuminuria and drop of glomerular filtration. In addition, we should consider that in patients with KD some drugs have proved a direct effect on the kidney, regardless of glycemia reduction. Other factors of the KD itself should be taken into account at the moment of treatment, such as major hydrosaline retention, cardiovascular risk, hypoglycemia risk, major half-life of drugs and fracture risk, among others.
\end{abstract}

Key words: kidney disease caused by diabetes, non-insulin treatment, insulin therapy.

Revista de la Sociedad Argentina de Diabetes 2017; Vol. 51 (94-99)
Especialista en Medicina Interna, especializada en Diabetes; Hospital de Clínicas José de San Martín; miembro del Comité de Nefropatía de la Sociedad Argentina de Diabetes, CABA, Argentina

Contacto de la autora: María Florencia Aranguren

E-mail: floraranguren@yahoo.com.ar
Correspondencia: Gral. Venancio Flores 93 (C1405CGA), CABA, Argentina

Tel: (011) 5991-0207

Fecha de trabajo recibido: 18/08/17

Fecha de trabajo aceptado: 30/08/17

Conflictos de interés: la autora declara que no existe

conflicto de interés

\section{Tratamiento farmacológico}

La enfermedad renal por diabetes (ERD) abarca una variedad de situaciones que hay que contemplar al momento de elegir el tratamiento.

Al inicio de la ER el control estricto de los factores de riesgo, incluido el de la glucemia, puede evitar su aparición y progresión. Por lo tanto en esta etapa hay que abocarse a cumplir con las metas glucémicas intensivas y preferentemente indicar drogas que disminuyan la microalbuminuria y la caída del filtrado glomerular (FG).

A medida que el $\mathrm{FG}$ cae, aumenta el riesgo de hipoglucemias, cuya presencia tiene efectos adversos cardiovasculares y conforman un factor de riesgo para la morbimortalidad car- diovascular, por lo cual hay que evitarlas. Por lo tanto, en etapas avanzadas de enfermedad renal elegir drogas que se asocien a menor riesgo de hipoglucemia es una buena alternativa'.

Por otro lado, esta patología compleja obliga a tener en cuenta otros factores propios de la ER que son determinantes al indicar el tratamiento farmacológico, como por ejemplo la mayor retención hidrosalina, el riesgo cardiovascular aumentado, el mayor riesgo de hipoglucemias, la mayor vida media de las drogas y el riesgo de fracturas, entre otros.

\section{Tratamiento no insulínico}

En la actualidad el tratamiento oral de la dia- 
betes mellitus tipo 2 (DM2) se basa, en la mayoría de las guías, en considerar a la metformina como la droga de elección de inicio, salvo que exista contraindicación para la misma. La principal contraindicación para la indicación de metformina es, sin dudas, el deterioro del FG. Si bien no existe un consenso unánime en cuanto al punto de corte para su suspensión, en los últimos años la mayoría de las guías concuerda en una mayor tolerancia para la suspensión de la metformina debido a que es una droga que ha demostrado efectos beneficiosos independientes del descenso de la glucemia, y por otro lado, el riesgo de acidosis láctica es bajo. En la Tabla 1 se resumen las indicaciones de suspensión según las diferentes entidades 1,2,3,4.

En el año 2016 la Food and Drugs Administration (FDA) actualizó la recomendación de suspensión de metformina en concordancia con las otras entidades, tomando en cuenta suspender con el filtrado menor de $30 \mathrm{ml} / \mathrm{min} / 1,73 \mathrm{~m}^{2}$, no aconseja iniciar entre $30-45 \mathrm{ml} / \mathrm{min} / 1,73 \mathrm{~m}^{2}$; si el paciente ya la estaba tomando, indicar la mitad de la dosis. Otras sugerencias de otras normas son obtener un filtrado antes de iniciar la droga, no iniciar la medicación con filtrado menor de $45 \mathrm{ml} / \mathrm{min} / 1,73 \mathrm{~m}^{2}$, repetir el FG anualmente o más frecuentemente a pacientes de riesgo como los ancianos, suspender la metformina antes de la administración de contrastes iodados con filtrado menor de $60 \mathrm{ml} / \mathrm{min} / 1,73 \mathrm{~m}^{2}$ y reiniciar luego de 48 hs tras obtener una confirmación analítica de estabilidad de la función renal ${ }^{3}$.

En la actualidad se encuentra en fase de investigación una formulación de metformina llamada Met DR, que presenta escasa absorción sistémica, con efecto predominante sobre el intestino. Probablemente esta nueva metformina podrá indicarse a pacientes con deterioro renal avanzado 5 .

Para la elección de la segunda droga antidiabética, en casos de no alcanzar las metas glucémicas con metformina o si la misma estuviera contraindicada, para individualizar el tratamiento debe considerarse una serie de factores ${ }^{1,6}$ :

- El riesgo de hipoglucemia.

- Si debe aumentar o bajar de peso.

- El riesgo cardiovascular.

- Las contraindicaciones según la farmacocinética o farmacodinamia de la droga.

- Los efectos adversos.

- El costo.

Además en pacientes con ER hay que considerar que algunas drogas han demostrado un efecto directo sobre el riñón, más allá del descenso de la glucemia, por ejemplo los inhibidores SGLT-2 (cotransportador de sodio-glucosa tipo 2) que reducen la reabsorción tubular de glucosa, la presión intraglomerular y disminuyen la albuminuria y la caída del FG7,8. Los agonistas del receptor de GLP-1 y los inhibidores de la DPP-4 también reportaron efectos beneficiosos sobre el riñón en diversos estudios ${ }^{9,10}$.

En la Tabla 2 se esquematizan todos los grupos de drogas antidiabéticas con respecto a los diferentes estadios de enfermedad renal. El color blanco significa que la droga puede ser utilizada, el gris oscuro con precaución y el negro que está contraindicada?.

\begin{tabular}{|c|c|c|c|c|}
\hline & Fabricante (prospecto) & $\begin{array}{l}\text { FDA Filtrado } \\
\mathrm{ml} / \mathrm{min} / \mathrm{m}^{2}\end{array}$ & Guías SAD & $\begin{array}{l}\text { KDOQI Y ADA Filtrado } \\
\mathrm{ml} / \mathrm{min} / \mathrm{m}^{2}\end{array}$ \\
\hline Función renal & $\begin{array}{l}\text { Contraindicación: } \\
\text { hombres: } \mathrm{Cr}>1,5 \mathrm{mg} / \mathrm{dl} \\
\text { mujeres: } \mathrm{Cr}>1,4 \mathrm{mg} / \mathrm{dl}\end{array}$ & $\begin{array}{l}\text { Contraindicación: } \\
<30 \text { no iniciar con menos de } \\
45 \text {. Si la toma, reducir dosis } \\
\text { a } 1 \mathrm{~g} / \text { día }\end{array}$ & $\begin{array}{l}\text { Contraindicación: } \\
\text { hombres: } \mathrm{Cr}>1,5 \mathrm{mg} / \mathrm{dl} \\
\text { mujeres: } \mathrm{Cr}>1,4 \mathrm{mg} / \mathrm{dl}\end{array}$ & $\begin{array}{l}\text { Precaución: } \\
\text { FG } 45 \text { a } 30 \text { dosis } \\
\text { máxima de } 1 \mathrm{~g} / \mathrm{d} \\
\text { suspensión <30 }\end{array}$ \\
\hline $\begin{array}{l}\text { Medio de } \\
\text { contraste }\end{array}$ & Suspender & Suspender & Suspender & $\begin{array}{l}\text { Suspender } 48 \mathrm{~h} \\
\text { antes y después }\end{array}$ \\
\hline
\end{tabular}

Cr: creatinina; Cl de Creat: clearance de creatinina; FG: filtrado glomerular; h: hora.

Tabla 1: Sugerencias extraídas de las guías de la Sociedad Argentina de Diabetes (SAD), Food and Drugs Administration (FDA), prospectos de la droga, normas National Kidney Foundation. KDOQI Clinical Practice Guideline for Diabetes and Chronic Kidney Disease (KDOOI) y guía de la American Diabetes Association (ADA), con respecto a la contraindicación o precaución en el uso de metformina. 


\begin{tabular}{|c|c|c|c|c|c|}
\hline & \multicolumn{4}{|c|}{ Clearance de creatinina $(\mathrm{ml} / \mathrm{min})$} & \multirow[b]{2}{*}{$<15$ ó dial } \\
\hline & $\geq 60$ & $60-45$ & $45-30$ & 30-15 & \\
\hline Metformina & & & & & \\
\hline Sulfonilureas & & & & $\begin{array}{l}\text { Glipizida y } \\
\text { gliclazida }\end{array}$ & \\
\hline Repaglinida & & & & & \\
\hline Pioglitazona & & & & & \\
\hline GLP-1 ag & & & & & \\
\hline (-) DPP-4 & & & & & \\
\hline (-) SGLT-2 & & $\begin{array}{l}\text { Canagliflozina } \\
\text { y empagliflozina }\end{array}$ & & & \\
\hline Insulina & & & & & \\
\hline
\end{tabular}

Tabla 2: El color blanco representa que la indicación del fármaco no merece mayor cuidado con respecto al filtrado; el color gris oscuro significa que debe tenerse precaución, ya sea por ser drogas capaces de producir hipoglucemias (como es el caso de la glipizida, gliclazida y repaglinida) o por generar retención hidrosalina y edemas (pioglitazona), etc.; el color negro indica que la droga o familia de drogas se encuentra contraindicada en el filtrado glomerular señalado. Adaptado de las normas KDOQI.

\section{Sulfonilureas y repaglinida}

La gliclazida y glipizida son las sulfonilureas (SU) de elección ante el deterioro del FG, dado que presentan una menor vida media y no generan metabolitos activos, condiciones que disminuyen el riesgo de hipoglucemia en comparación con el resto de las SU. De hecho algunos trabajos indican que la gliclazida MR presenta aproximadamente un 50\% menos de riesgo de hipoglucemias que la glimepirida ${ }^{11}$. Incluso otros estudios evidenciaron que la gliclazida no presenta un riesgo significativamente mayor de hipoglucemias que los inhibidores de la DPP-4 (dipeptidilpeptidasa-4) ${ }^{12}$. Otro punto a favor de esta droga es que algunos trabajos evidenciaron que el riesgo de mortalidad cardiovascular es menor con gliclazida que con otras SU ${ }^{13}$. Cabe destacar, entonces, que la gliclazida constituye una buena opción dentro de la familia de las SU para el tratamiento de los pacientes con ER.

La repaglinida también es una opción válida sobre todo en casos de hiperglucemia postprandial.

Las normas Kidney Disease Outcomes Quality Initiative de la National Kidney Foundation (KDOQi) avalan la indicación de la glipizida, gliclazida y repaglinida para todos los estadios de ER. Sin embargo, la precaución a tener en cuenta en estos casos es el riesgo de hipoglucemias, por lo que debe considerarse disminuir la dosis a medida que el filtrado cae como una medida de prevención". En la Tabla 3 se resumen las principales características farmacocinéticas de las SU y las recomendaciones de las normas KDOQI y de la ADA con respecto a la indicación o suspensión para pacientes con ER ${ }^{11,14}$. 


\begin{tabular}{|l|l|l|l|l|}
\hline \multicolumn{1}{|c|}{ Droga } & \multicolumn{1}{|c|}{ Vida media } & \multicolumn{1}{|c|}{$\begin{array}{c}\text { Vida media de los } \\
\text { metabolitos activos }\end{array}$} & \multicolumn{1}{c|}{ Excreción } & \multicolumn{1}{c|}{$\begin{array}{c}\text { Recomendación } \\
\text { KDOQI y ADA }\end{array}$} \\
\hline Glibenclamida & $5-7 \mathrm{hs}$ & $\begin{array}{l}10 \mathrm{hs} \text { metabolito } \\
\text { activo }\end{array}$ & $50 \%$ renal & $\begin{array}{l}\text { Suspender con FG } \\
<\text { de } 60\end{array}$ \\
\hline Glimepirida & $5-8 \mathrm{hs}$ & Activo 3-6 hs & $80 \%$ renal & $\begin{array}{l}\text { Utilizar con dosis } \\
\text { bajas y precaución de } \\
\text { hipoglucemias }\end{array}$ \\
\hline Glipizida & $2-4 \mathrm{hs}$ & Inactivo & $70 \%$ renal & $\begin{array}{l}\text { Precaución de hipo- } \\
\text { glucemias }\end{array}$ \\
\hline Gliclazida & $10 \mathrm{hs}$ & Inactivo & $65 \%$ renal & $\begin{array}{l}\text { Precaución de hipo- } \\
\text { glucemias }\end{array}$ \\
\hline
\end{tabular}

Tabla 3: Farmacocinética de las sulfonilureas y recomendaciones para pacientes con enfermedad renal según la American Diabetes Association (ADA) ${ }^{13}$ y las normas National Kidney Foundation. KDOQI Clinical Practice Guideline for Diabetes and Chronic Kidney Disease (KDOQI) 1.

\section{Pioglitazona}

Esta tiazolidindiona no presenta contraindicación por FG en ningún estadio de disfunción renal. Sin embargo, cabe tener en cuenta que provoca demostrada retención hidrosalina, edemas, insuficiencia cardíaca congestiva y fracturas, efectos adversos que serán más peligrosos cuanto menor sea el filtrado del paciente?.

\section{Drogas relacionadas con incretinas}

En este ítem se mencionan dos grupos de drogas: los agonistas del receptor de GLP-1 (g/ucagon like peptide) y los inhibidores de DPP-4. Las primeras son exenatide y liraglutide, drogas inyectables, ambas contraindicadas con filtrado menor de $30 \mathrm{ml} / \mathrm{min} / 1,73 \mathrm{~m}^{2}$. Dado que son drogas que como efecto adverso causan náuseas y vómitos hay que tener en cuenta que los pacientes que las consumen pueden presentar algún grado de deshidratación si no son alertados al indicarlas. El estudio LIRA-RENAL evidenció seguridad en la administración de liraglutide para pacientes con FG entre 30 y $60 \mathrm{ml} / \mathrm{min} / 1,73 \mathrm{~m}^{2}$, sin alterar la función renal ni producir más hipoglucemias que en el grupo placebo ${ }^{15}$. Si se tiene en cuenta que los pacientes en este estadio de ER presentan una elevada incidencia de eventos cardiovasculares y el estudio Liraglutide Effect and Action in Diabetes: Evaluation of Cardiovascular Outcome Results (LEADER) mostró una disminución de los mismos ${ }^{16}$, este análogo del GLP-1 es una buena alternativa para pacientes con FG hasta $30 \mathrm{ml} / \mathrm{min} / 1,73 \mathrm{~m}^{2}$.

Los inhibidores de DPP-4 pueden indicarse en todos los estadios de enfermedad renal, sólo que algunos de ellos requieren ajuste de dosis según el filtrado. En la Tabla 4 se resu-

\begin{tabular}{|c|c|c|c|}
\hline Drogas & $\begin{array}{l}\mathrm{FG}>50 \\
\mathrm{ml} / \mathrm{min}\end{array}$ & $\begin{array}{c}\text { FG } 30-50 \\
\mathrm{ml} / \mathrm{min}\end{array}$ & $\begin{array}{c}\text { FG }<30 \mathrm{ml} / \mathrm{min} \\
\text { hemodiálisis }\end{array}$ \\
\hline Exenatide & $\begin{array}{l}5 \mu \mathrm{g} \mathrm{sc} \times 2 \\
60 \text { min antes almuerzo y cena. } \\
\text { Mantenimiento }=10 \mu \mathrm{g} \mathrm{sc}\end{array}$ & \multirow{2}{*}{ No se ajustan } & \multirow{2}{*}{ Contraindicado } \\
\hline Liraglutide & $\begin{array}{l}0,6 \mathrm{mg} \text { sc sin relación/comida. } \\
\text { Mantenimiento }=1,2-1,8 \mathrm{mg}\end{array}$ & & \\
\hline Sitagliptina & 100 mg. Sin relación/comida & $50 \mathrm{mg}$ & $\begin{array}{l}25 \mathrm{mg} \text {. No se remueve } \\
\text { x HD }\end{array}$ \\
\hline Saxagliptina & 2,5-5 mg. Sin relación/comida & $2,5 \mathrm{mg}$ & 2.5. Removido $\times \mathrm{HD}$ \\
\hline Vildagliptina & 50 mg dos veces por día & 50 mg/día & 50 mg/día \\
\hline Teneligliptina & & \multicolumn{2}{|c|}{20 mg. No modifica dosis } \\
\hline Linagliptina & & \multicolumn{2}{|c|}{$5 \mathrm{mg}$. No modifica dosis } \\
\hline
\end{tabular}

HD: hemodiálisis.

Tabla 4: Ajuste según el filtrado glomerular de las drogas relacionadas con incretinas. Adaptado de las guías de la National Kidney Fundation 2012. 
men los ajustes de las drogas relacionadas con incretinas?

\section{Inhibidores de SGLT-2}

Este grupo de drogas es novedoso desde el punto de vista de su mecanismo de acción y también porque presenta efectos beneficiosos independientemente de la disminución de la glucemia, por ejemplo, la reducción del hiperfiltrado glomerular, la microalbuminuria y la mortalidad cardiovascular, en el último de los casos en pacientes con enfermedad cardiovascular previa?. Por lo tanto son una excelente elección para estadios iniciales de enfermedad renal, sin embargo no para estadios avanzados. La dapagliflozina se encuentra contraindicada en pacientes con filtrado menor de $60 \mathrm{ml} / \mathrm{min} / 1,73 \mathrm{~m}^{2}$, y la canagliflozina y empagliflozina con filtrado menor de $45 \mathrm{ml} / \mathrm{min} / 1,73 \mathrm{~m}^{2}$. Ambas requieren ajuste de la dosis entre 45 y 60 de FG ${ }^{14}$. Debe tenerse en cuenta a la hora de indicarlas en pacientes con ER establecida que existe reporte de aumento de la tasa de fracturas con la utilización de estas drogas.

A modo de síntesis, y teniendo en cuenta un enfoque clínico, cabe resaltar que la elección de los fármacos no insulínicos son una buena opción para pacientes con hiperglucemias moderadas o leves. Aquellos con hiperglucemias severas deben ser insulinizados. Por otro lado, debe tenerse en cuenta el peso del paciente; por ejemplo los inhibidores de DPP-4 son una excelente alternativa para aquellos con sobrepeso en todos los estadios de enfermedad renal por su neutralidad o GLP-1 por disminuir el mismo, sin embargo, ante casos de desnutrición o pérdida de peso el tratamiento de elección debe ser la insulina sin dudas.

\section{Insulinoterapia}

No existen contraindicaciones relacionadas con la indicación de ninguna insulina en particular con respecto al filtrado renal.

Un punto de interés es que las nuevas insulinas, con vida media más prolongada como ser glargina U-300 ó degludec, evidenciaron según algunos estudios una menor tasa de hipoglucemias, incluyendo a los pacientes con deterioro del filtrado ${ }^{17}$.

La indicación de una u otra insulina debe guiarse por el monitoreo, las características del paciente y el conocimiento de los perfiles farmacocinéticos de las moléculas.

Para aquellos con DM1 la insulinoterapia de elección debe ser el tratamiento intensificado, mediante el cual el paciente coloca una o más dosis de insulina basal (NPH, detemir, glargina o degludec), más correcciones que deben realizarse con insulinas preferentemente ultrarrápidas dada su menor tasa de hipoglucemias en comparación con la regular o corriente. Los análogos de insulina ultrarrápidos disponibles son glulisina, lispro y aspártica. La corrección con los mismos debe efectuarse en base a la glucemia que presenta el paciente más el conteo de los hidratos de carbono que ingerirá.

Para pacientes con DM2 la insulinoterapia puede indicarse en cualquier momento de la enfermedad, en forma transitoria o permanente, asociada a otros agentes o con un esquema intensificado, de acuerdo a las características del individuo.

Con respecto a la insulinoterapia del paciente que recibe tratamiento renal sustitutivo, cabe considerar que la dosis de insulina puede variar entre el día de diálisis y el día sin la misma. Otro punto a tener en cuenta es el horario de colocación y se aconseja que la insulina basal se administre después del turno de diálisis. Sin embargo, como en la mayoría de los casos, debe ser el monitoreo glucémico el que guíe el tratamiento individualizado de estos pacientes.

\section{BIBLIOGRAFÍA}

1. National Kidney Foundation. KDOQI Clinical practice guideline for diabetes and CKD: 2012 update. Am J Kidney Dis 2012; 60(5):850-886.

2. American Diabetes Association. Standards of Medical Care in Diabetes 2017. Diabetes Care 2017; 40 (Suppl 1). 
3. Metformin-containing drugs: Drug Safety Communication. Revised warnings for certain patients with reduced kidney function https://www.fda.gov/Safety/MedWatch/ Safetylnformation/SafetyAlertsforHumanMedicalProducts/ucm 494829.htm?source5govdelivery\&utm medium5email\&utm_source5govdelivery.revisado 20/03/2017

4. Frechtel G, Litwak L, et al. Guía del tratamiento de la diabetes mellitus tipo 2. Sociedad Argentina de Diabetes. Revista de la Sociedad Argentina de Diabetes 2010; 44 (5): 366-367.

5. Buse JB, DeFronzo RA, Rosenstock J, et al. The primary glucose-lowering effect of metformin resides in the gut, not the circulation: results from short-term pharmacokinetic and 12-week dose-ranging studies. Diabetes Care 2016; 39(2):198-205.

6. Davies M, Chatterjee S, Khunti K. The treatment of type 2 diabetes in the presence of renal impairment: what we should know about newer therapies. Clinical Pharmacology: Advances and Applications 2016; 8: 61-81.

7. Cherney DZI, Perkins BA, Soleymanlou N, et al. Renal hemodynamic effect of sodium-glucose cotransporter 2 inhibition in patients with type 1 diabetes mellitus. Circulation 2014; 129(5): 587-597.

8. Heerspink HJL, Desai M, Jardine M, et al. Canagliflozin slows progression of renal function decline independently of glycemic effects. J Am Soc Nephrol 2017; 28:368-375.

9. Marso SP, Daniels GH, Brown-Frandsen K, et al. Liraglutide and cardiovascular outcomes in type 2 diabetes. $\mathrm{N}$ Engl J Med 2016; 375(4):311-22.
10. Cooper ME, Perkovic V, McGill JB, et al. Kidney disease end points in a pooled analysis of individual patient-level data froma large clinical trials program of the dipeptidyl peptidase 4 inhibitor linagliptin in type 2 diabetes. Am J Kidney Dis 2015; 66(3):441-9.

11. Sola D, Rossi L, Schianca GPC, et al. Sulfonylureas and their use in clinical practice. AMS 2015; 11(4): 840-848.

12. Mbanya JC, Al-Sifiri S, Abdel-Rahim A, et al. Incidence of hypoglycemia in patients with type 2 diabetes treated with gliclazide versus DPP-4 inhibitors during Ramadan: A meta-analytical approach. Diabetes Res Clin Pract 2015 Aug; 109(2):226-32.

13. Monami M, Balzi D, Lamanna C, et al. Are sulphonylureas all the same? A cohort study on cardiovascular and cancer-related mortality. Diabetes Metab Res Rev 2007 Sep; 23(6):479-84.

14. Tuttle K. Bakris GL, Bilous RW, et al. Diabetic kidney disease: a report from an ADA Consensus Conference. Diabetes Care 2014; 37:2864-2883.

15. Davies M, Bain SC, Atkin SL, et al. Efficacy and safety of liraglutide vs placebo as add-on to glucose-lowering therapy in patients with type 2 diabetes and moderate renal impairment (LIRA-RENAL): a randomized clinical trial. Diabetes Care 2016; 39(2):222-230.

16. Marso S, Daniels GH, Brown-Frandsen K, et al. Liraglutide and cardiovascular outcomes in type 2 diabetes. $\mathrm{N}$ Engl J Med 2016 July 28; 375(4): 311-322.

17. Senior P, Escalada J, Halimi S, et al. Glycemic control and hypoglycemia benefits with insulin glargine $300 \mathrm{U} / \mathrm{mL}$ (Gla-300) extend to people with type 2 diabetes and mild-to-moderate renal impairment. Canadian Journal of Diabetes 2016; 40(5): S47. 